1999-01-01

\title{
Experimental Observation of Individual Single Wall Nanotube Species by Raman Microscopy
}

\author{
G. S. Duesberg \\ Trinity College Dublin \\ W. Blau \\ Technological University Dublin \\ Hugh Byrne \\ Technological University Dublin, hugh.byrne@tudublin.ie
}

See next page for additional authors

Follow this and additional works at: https://arrow.tudublin.ie/nanolart

Part of the Physics Commons

\section{Recommended Citation}

Duesberg, G. et al. (1999) Experimental observation of individual single wall nanotube species by Raman microscopy. Chemical Physics Letters, Volume 310, Issues 1-2, Pages 8-14. 27 August. doi: 10.21427/ wn5b-zs78

This Article is brought to you for free and open access by the NanoLab at ARROW@TU Dublin. It has been accepted for inclusion in Articles by an authorized administrator of ARROW@TU Dublin. For more information, please contact arrow.admin@tudublin.ie, aisling.coyne@tudublin.ie,gerard.connolly@tudublin.ie.

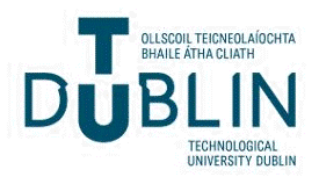


Authors

G. S. Duesberg, W. Blau, Hugh Byrne, J. Muster, M. Burghard, and S. Roth

This article is available at ARROW@TU Dublin: https://arrow.tudublin.ie/nanolart/15 


\title{
Experimental observation of individual single wall nanotube species by Raman microscopy
}

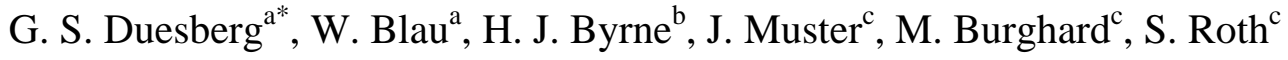 \\ ${ }^{\text {a }}$ Physics Dept., Trinity College Dublin, Dublin 2, Ireland \\ ${ }^{\mathrm{b}}$ School of Physics, Dublin Institute of Technology, Kevin Street, Dublin 8, Ireland \\ c Max-Planck-Institut fuer Festkoerperforschung, Heisenbergstr. 1, 70569 Stuttgart, \\ Germany
}

\begin{abstract}
Individual single wall carbon nanotubes (SWNTs) or a small rope of SWNTs with the same diameter have been characterised by Raman spectroscopy. Highly pure, length selected SWNTs adsorbed onto substrates designed for surface enhanced Raman Spectroscopy were investigated with a Raman microscope. Various spectra, each with a distinct sharp peak in the radial breathing mode area and four C-C stretching modes could be observed. The modes also showed a strong variation in the relative intensities. In correlation with theoretical predictions this should establish Raman microscopy as a non destructive nano-technological tool, capable of determining the diameter, symmetry and position of individual SWNTs.
\end{abstract}

*corresponding author, E-mail: duesberg@klizix.mpi-stuttgart.mpg.de, Fax: ++49-711-689-1010 


\section{Introduction}

A vast range of potential applications [1], as well as fundamental research have raised much interest in carbon nanotubes. One of the most promising applications lies in the design of electric and mechanical nanoscale devices [2, 3]. For those applications, very pure and well characterised nanotubes are essential. Since common production methods always lead to a variety of carbon species and different nanotubes, the purification and characterisation have become a crucial issue in carbon nanotube science. The introduction of chromatographic methods has made it possible to purify and length separate carbon nanotubes [4], thus facilitating studies of well defined materials.

Raman spectroscopy has become a powerful tool for the characterisation of nanotubes. For different SWNTs, defined in diameter and chirality by their roll-up vector $(n, m)$, variations in the Raman spectra have been calculated $[1,5,6]$. In the radial breathing mode (RBM), the carbon atoms undergo an uniform radial displacement. This low frequency mode is centred around $200 \mathrm{~cm}^{-1}$ for typical SWNT samples. Experimental data confirmed that the diameter of a SWNT can directly be estimated from the frequency of this single low frequency mode [7]. Several C-C bond motions contribute to G-line of SWNTs centred at $1580 \mathrm{~cm}^{-1}$, which only weakly depend on diameter and helicity of the SWNT [1]. For example, the split of $E_{2 g}$ mode of graphite $\left(1582 \mathrm{~cm}^{-1}\right)$ due to the introduction of curvature into a graphene sheet increases with decreasing diameter [8]. A single tube is predicted therefore to have a single low frequency mode, with a multiplet of modes in the $1580 \mathrm{~cm}^{-1}$ region. The disordered carbon line (D-line) around $1350 \mathrm{~cm}^{-1}$ line is usually assigned to carbon impurities, which are present in bulk samples. Even though calculations 
showed that SWNTs also have a weak contribution in this region, the D-line has been used for estimation of sample purity [9, 10].

Using different laser lines for excitation, specific tubes are resonantly enhanced to various degrees [11]. This is a result of the unique 1D structure of the SWNTs which gives rise to van Hove singularities in their density of states. The energy gap between the first van Hove singularities depends on the chirality and diameter of a SWNT. In this way, the metallic or semiconducting nature of a SWNT can be distinguished, because their resonant enhancement conditions are significantly different [12]. In addition, it has been observed that the G-line modes of metallic tubes are broadened and downshifted with respect to semiconducting SWNTs [13].

The relative intensities and polarisation of the scattered modes vary with the orientation of nanotubes with respect to the polarisation of the laser line $[6,14]$, thus the orientation of the tubes could be estimated with polarisation dependent Raman spectroscopy. Furthermore, mechanical properties of nanotubes can be investigated by Raman spectroscopy [15].

Until now, however, most of the Raman investigations have been performed on samples containing a variety of non-aligned SWNTs. The spectra obtained are always a superposition of various species of tubes, causing broad unresolved peaks. This makes a straight forward assignment of the G-line modes in bulk samples impossible [16]. In the low frequency area, the diameter distribution in the sample is reflected by the complexity of the spectra, but a quantitative analysis is difficult because of wavelength dependent resonant Raman effects. Consequently, the Raman spectra change significantly with the energy of the incident laser for a given bulk sample, because the contribution of the resonantly enhanced SWNTs are dominant [17]. 
In this letter, we present Raman spectra originating from individual SWNTs or small ropes of SWNTs. Using aqueous surfactant suspensions, obtained by chromatographic separation, it is possible to adsorb the NTs in a controlled way on substrates for surface enhanced Raman spectroscopy (SERS). The rough silver substrates were then investigated with a Raman microscope. Scanning Electron and Atomic Force Microscopy (SEM, AFM) and investigations revealed that there is only a limited number of tubes in the area of the incident beam. Spectra with single RBM-modes and a well defined G-line multiplets are presented.

\section{Experimental}

The SWNTs were produced by the arc discharge method with $\mathrm{Ni} / \mathrm{Y}$ catalysts [18]. The raw material (5 mg) was added to $5 \mathrm{ml}$ of aqueous $1 \mathrm{wt} \%$ sodium dodecylsulfate solution and sonicated for $3 \mathrm{~min}$ with an ultrasonic tip. The obtained stable suspension was subjected to chromatography as described previously [19]. The purified and length separated SWNTs were applied onto silver substrates produced by the reduction of Tollen's reagent as described by Ni et al. for SERS applications [20]. This method produces highly reproducible substrates with colloidal features of the order of 40-150nm yielding an enhancement of $\sim 1 \mathrm{x} 10^{5}$. The adsorption times of the SWNTs were varied form 30 sec to $30 \mathrm{~min}$, followed by the immersion into distilled water to remove the surfactant. For comparison, SWNTs were also adsorbed onto cleaned silicon wafers and glass slides, treated for 15 min with an aqueous solution of 3-aminopropyltriethoxylsilane (APS, $2.5 \mathrm{mM}$ ) as previously described [21]. The samples were characterised with AFM (Digital Instruments, Nanoscope IIIa) in Tapping mode and a field emission SEM (Gemini DSM 982). 
The Raman microscope was an Instruments SA Labspec 1B, equipped with a $20 \mathrm{~mW}$ He/Ne laser with a emission at $632.8 \mathrm{~nm}$. Previous studies on chromatographically separated SWNT suspensions illustrate that there is a strong enhancement of metallic tubes at this wavelength $[12,25]$. The microscope had a manual $\mathrm{X}-\mathrm{Y}$ stage and the spot of the incident laser beam is specified to be $1 \mu \mathrm{m}$ in diameter. This leads to intensities approximately $10^{6} \mathrm{~W} / \mathrm{cm}^{2}$ in the Raman active area. A grating of 1800 lines/mm was employed yielding a resolution of $1.5 \mathrm{~cm}^{-1}$ per pixel. The spectra were recorded under ambient conditions by focusing the laser spot on the surface of the substrates.

\section{Results}

Unlike other purification techniques, chromatographic separation yields individual SWNTs and small ropes of 3-5 SWNTs in aqueous suspension. Approximately 50\% of the objects are individual tubes while the other half are ropes. This was estimated by the analysis of height profiles taken with the AFM of the suspensions adsorbed on silicon wafers [19].

In Fig. 1 a SEM image of a typical SERS substrate used for Raman investigations is shown. The rough surface structure are of silver dots of approximately $100 \mathrm{~nm}$ in diameter. A few, spatially separated SWNTs or ropes of SWNTs can be seen on the surface. Hence, the use of SERS substrates made it possible to detect small numbers of tubes, using a Raman microscope, which has a spot size of approximately $1 \mu \mathrm{m}^{2}$. Short acquisition times of the Raman spectra could also be employed minimising perturbations like drift of the substrate. The exact magnitude of the surface enhancement for tubes by the silver substrates was difficult to determine. Variations in intensity for different areas of one substrate can result from different types of tubes 
or even different orientation of the tube with respect to the laser polarisation. Unfortunately some of the substrates were not stable under exposure to the laser beam, this instability being clearly observable as large fluctuating features in the spectrum. For stable substrates the enhancement factor is up to 50000 with respect to a comparable glass substrate.

Various spots on SERS substrates with low surface coverage exhibited no observable signal, while on others strong SWNT modes could be collected. The spectra usually showed a few or even a single very sharp peak in the RBM region, as well as resolved peaks around $1580 \mathrm{~cm}^{-1}$. A broad feature at $1270 \mathrm{~cm}^{-1}$, whose intensity correlated well with the intensity of the G-line was also apparent. A spectrum with two distinct features in the low frequency area is shown in Fig. 2. A fit of three Lorentzians centred at 166, 191 and $196 \mathrm{~cm}^{-1}$ with full width at half maximums (FWHMs) less than $10 \mathrm{~cm}^{-1}$ is shown in the inset. The G-line around $1580 \mathrm{~cm}^{-1}$ exhibits distinct features but is not clearly resolved.

At various spots, spectra with a single peak in the low frequency area could be obtained. As an example, a spectrum with a single line at $165 \mathrm{~cm}^{-1}$ and four peaks at 1518, 1540, 1563, and $1590 \mathrm{~cm}^{-1}$, is displayed in Fig. 3 (the sharp line at $181 \mathrm{~cm}^{-1}$ is an instrumental artefact). In Table 1, the line positions and width of spectra exhibiting a single RBM are summarised. For the Lorentzians - shaped RBMs linewidths between 6 - $10 \mathrm{~cm}^{-1}$ were obtained. The G-line could be fitted by four Lorentzians between 1510 - $1595 \mathrm{~cm}^{-1}$ with FWHMs smaller than $30 \mathrm{~cm}^{-1}$. It is important to note that the relative intensities of the low frequency RBM and the G-line modes differed dramatically in spectra taken in different spots.

\section{Discussion}


SEM and AFM investigations of the substrates reveal that there is on average only a small number objects are present in an area of $1 \mu \mathrm{m}^{2}$. Since the laser spot of the Raman microscope approximately $1 \mu \mathrm{m}$ in diameter, the spectra originate from a few SWNTs. In contrast to bulk samples, where the spectra are a superposition of numerous signals from various tubes resulting in broad unresolvable features, distinct sharp peaks in the low frequency range and a resolvable splitting of the G-line are observed. The Lorentzian line shape of the RBM also indicates that the scattering originates from single objects. The obtained FWHMs below $10 \mathrm{~cm}^{-1}$ are consistent with the expected natural linewidth of this mode because of the electron phonon broadening in graphite [22, 23]. In previous works on bulk samples, up to ten Lorentzians with FWHMs smaller than $10 \mathrm{~cm}^{-1}$ had to be used to fit the broad low frequency signals.

The RBM is directly related to the diameter of the tube by the relation:

$\mathrm{d}=\frac{223.75}{\omega}[7]$

were $d$ is the diameter of the given tube in nanometers and $\omega$ the $R B M$ in wavenumbers. Assuming a resolution of $5 \mathrm{~cm}^{-1}$ the diameter of a tube can be derived with an accuracy of approximately $\pm 0.05 \mathrm{~nm}$. The results are listed in Table 1 . However, it has to be considered that a recent work shows deformations of adsorbed nanotubes due to interaction with the surface [24]. Therefore, an influence of the substrate or of surrounding tubes, e.g. the difference between an individual tube or a rope, on the position of the RBM can not be ruled out.

The diameter dependence of the G-line modes is approximately an order of magnitude smaller than for the RBM, which causes overlaying peaks in bulk samples. Therefore a correlation between the RBM and the G-line modes was until now impossible. In 
Table 1 the positions and line widths of the four G-line modes which correspond to a single RBM are given. They could be fitted with four Lorentzians exhibiting FWHMs below $30 \mathrm{~cm}^{-1}$. The linewidth and frequencies match the results of Pimenta et al. who reported broadened and downshifted G-line modes for metallic SWNTs. Only the position of the highest frequency mode does not appear to be downshifted. Selective resonant enhancement of metallic SWNTs of diameters between 1.1 - $1.6 \mathrm{~nm}$ has been reported using red laser light [12]. This is because the energy gap between the first van Hove singularities of these SWNTs is similar to the photon energy. Indeed in chromatographically separated SWNT suspensions, a progressive variation in the degree of enhancement can be correlated with tube length [25]. For the purpose of this experiment, therefore, the observed spectra may be presumed to originate from metallic SWNTs.

Using this assumption, the possible $(n, m)$ combinations given by

$\mathrm{d}=0.079 \sqrt{\mathrm{m}^{2}+\mathrm{nm}+\mathrm{n}^{2}}$

can be restricted to those of metallic SWNTs. The criterion for a SWNT to be metallic is:

$\frac{\mathrm{n}-\mathrm{m}}{3}=$ int eger $[1]$

Therefore, in Table 1 only $(n, m)$ combinations satisfying these conditions are considered.

The change in relative intensities of the RBM relative to the G-line can be explained with calculations performed by Saito et al. [8], who showed that the polarisation and intensity of Raman scattered light depends on the position of the tube with respect to the polarisation of the incident laser beam. In addition, the modes of zigzag, armchair and chiral SWNTs do not show exactly the same behaviour. Since the position of the 
SWNTs on the substrate is arbitrary, each spectrum should show a different intensity distribution of the different modes. Exactly this has been observed: The relative intensities of the different modes with respect to each other changes dramatically in the recorded spectra. It has also been observed that lines vanished after introducing an analyser in front of the detector [25]. In this respect it has to be noted that the surface enhancement can change intensity, symmetry and even position of mode selectively. The origin of the intense peak at $1270 \mathrm{~cm}^{-1}$ can not be explained by the presence of impurities, which are removed by purification, even though a residue of amorphous carbon attached to SWNTs may still remain. It is more likely that the peak originates from the same object as the G-line since the intensities are directly correlated. A selective surface enhancement of the rather weak Raman modes calculated in this region could take place. Another tentative explanation is that modes in this region are induced by symmetry breaking effects such as bending or the interaction with the substrate [26].

\section{Conclusion}

Raman spectra of a small number of SWNTs or of individual SWNTs are presented. Some spectra, taken on SWNTs adsorbed onto SERS substrates, showed a single RBM and well defined modes at the G-line. From the position of the RBM the diameter of the tube was derived. Since there is evidence that the spectra originate from metallic SWNTs, the spectra could be assigned to only a few possible role up vectors defining the tube.

More detailed investigations with for example, various laser lines and polarisation dependent Raman microscopy should allow to assign the modes of a spectrum or to 
estimate the orientation of the tube relative to the polarisation of the laser line. The role of the surface-tube interaction also has to be clarified.

\section{Acknowledgements}

We gratefully thank C. Journet (Univ. of Montpellier, France) for the SWNT samples. This work has been supported by the EU TMR network NAMITECH, ERBFMRX-CT96-0067 (DG-MIHT).

\section{Figure captions:}

Fig. 1: A SEM image of the Tapping mode of a SERS substrate. The image shows a few SWNTs or ropes of SWNTs in an area of approximately $1 \mu \mathrm{m}^{2}$.

Fig. 2: A Raman spectrum recorded on a SERS substrate. The inset shows three lines in the low frequency range, each corresponding to SWNTs with different diameters. The G-line has distinct feature, but is not clearly resolved.

Fig. 3: Raman spectrum recorded on a SERS substrate. The insets show a single RBM at $165 \mathrm{~cm}^{-1}$, and four lines around $1580 \mathrm{~cm}^{-1}$. The feature at $181 \mathrm{~cm}^{-1}$ is an instrumental artefact. 


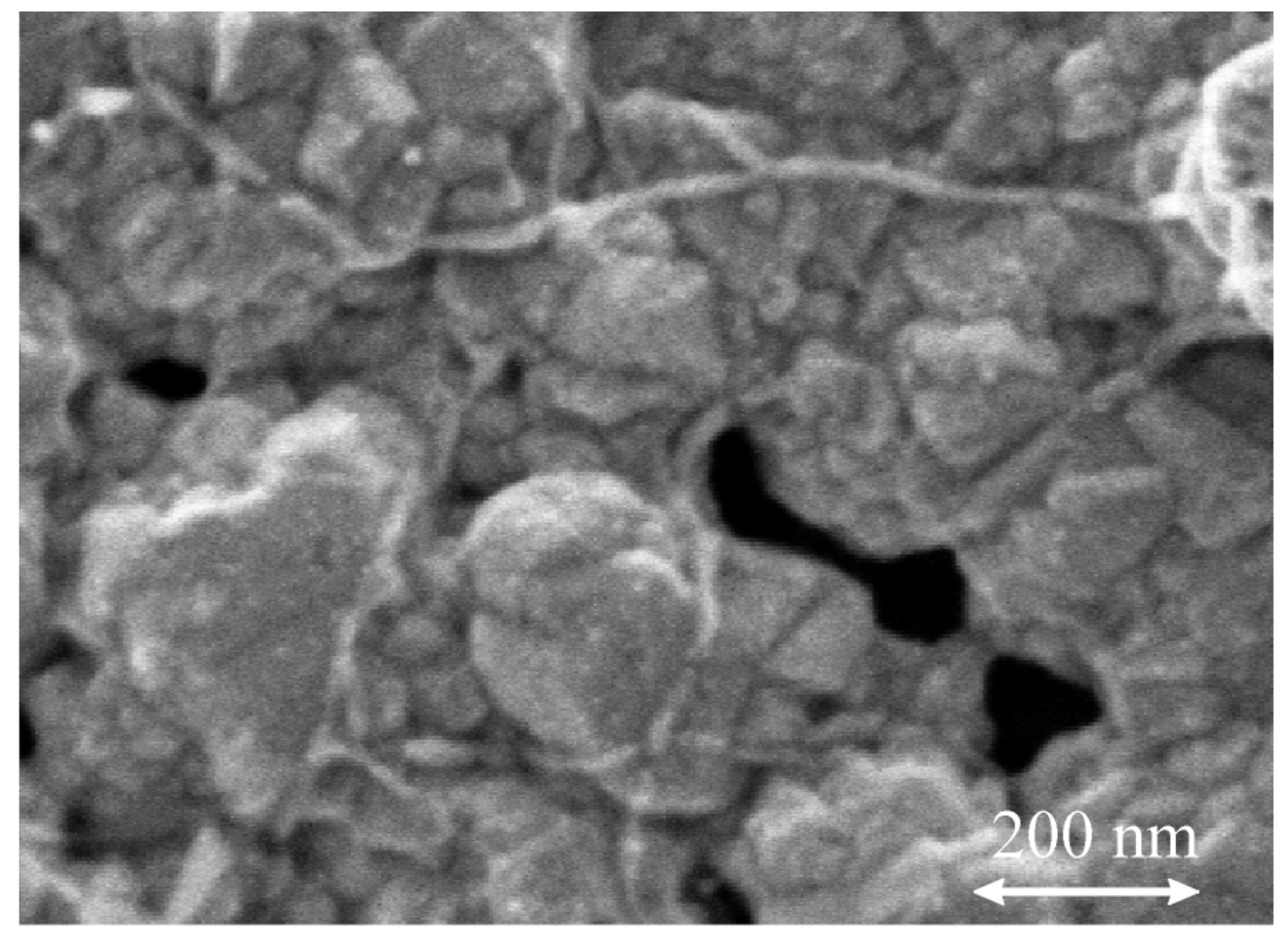

Fig. 1 


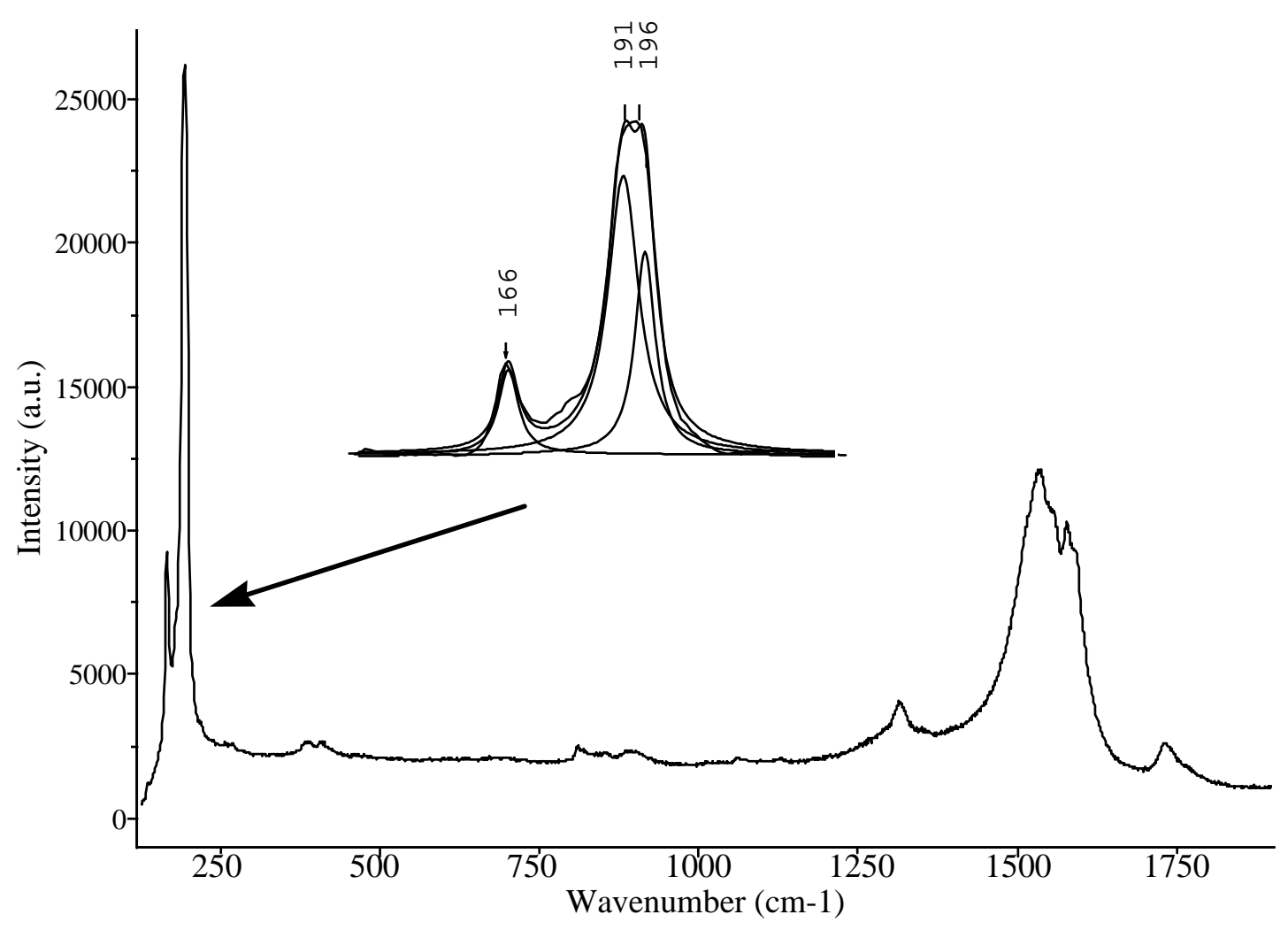

Fig. 2 


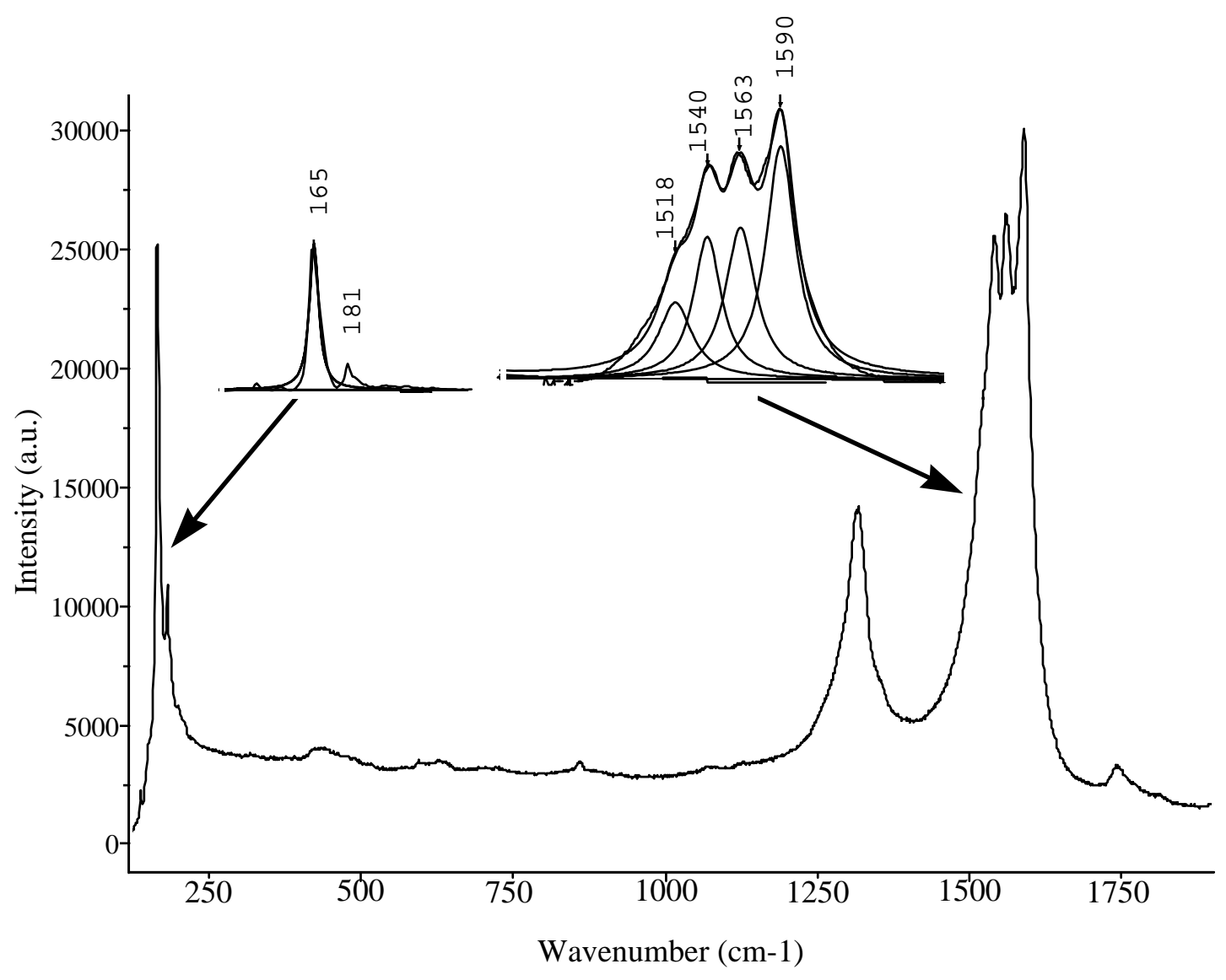

Fig. 3 


\begin{tabular}{lccccccccccl}
\hline $\begin{array}{l}\text { RBM } \\
\text { /cm-1 }\end{array}$ & $\begin{array}{c}\text { FWHM } \\
\text { /cm-1 }\end{array}$ & $\begin{array}{c}\text { G1/ } \\
\text { cm-1 }\end{array}$ & $\begin{array}{c}\text { FWHM } \\
\text { /cm-1 }\end{array}$ & $\begin{array}{c}\text { G2/ } \\
\text { cm-1 }\end{array}$ & $\begin{array}{c}\text { FWHM } \\
\text { /cm-1 }\end{array}$ & $\begin{array}{c}\text { G3/ } \\
\text { cm-1 }\end{array}$ & $\begin{array}{c}\text { FWHM } \\
\text { /cm-1 }\end{array}$ & $\begin{array}{c}\text { G4/ } \\
\text { cm-1 }\end{array}$ & $\begin{array}{c}\text { FWHM } \\
\text { /cm-1 }\end{array}$ & $\begin{array}{l}\text { d } \\
\text { /nm }\end{array}$ & possible (n,m) \\
\hline 165 & 6 & 1518 & 28 & 1540 & 23 & 1563 & 25 & 1590 & 25 & 1.36 & $(10,10)(13,7)(14,5)$ \\
172 & 9 & 1525 & 15 & 1542 & 16 & 1564 & 12 & 1592 & 27 & 1.30 & $(11,8)(15,3)(16,1)$ \\
173 & 9 & 1526 & 10 & 1542 & 18 & 1563 & 29 & 1592 & 26 & 1.30 & $(11,8)(15,3)(16,1)$ \\
191 & 8 & 1520 & 25 & 1540 & 26 & 1562 & 29 & 1592 & 25 & 1.17 & $(15,0)(10,7)(14,2)$ \\
192 & 9 & 1512 & 18 & 1535 & 27 & 1561 & 26 & 1591 & 29 & 1.17 & $(15,0)(10,7)(14,2)$ \\
196 & 9 & 1524 & 23 & 1543 & 24 & 1565 & 27 & 1590 & 28 & 1.14 & $(10,7)$ \\
199 & 7 & 1516 & 24 & 1540 & 29 & 1568 & 27 & 1592 & 20 & 1.12 & $(11,5)$ \\
200 & 10 & 1516 & 25 & 1538 & 24 & 1565 & 20 & 1595 & 22 & 1.12 & $(11,5)$ \\
202 & 7 & 1526 & 20 & 1544 & 18 & 1568 & 24 & 1595 & 27 & 1.11 & $(8,8)(11,5)$ \\
\hline
\end{tabular}

\section{Table 1}

Table 1: The table presents the positions and line width of nine sample spectra obtained from SERS substrates. Each spectrum features only one RBM, fitted with a single Lorentzian. The spectra are listed in order of increasing RBM frequency. The high frequency modes were fitted with four Lorentzian, G1 - G4. The estimated diameter and a tentative assignment to specific SWNTs are given in the last two columns. 


\section{References}

${ }^{1}$ M.S. Dresselhaus, G. Dresselhaus, P.C. Eklund, Science of Fullerenes and Carbon Nanotubes, Academic Press, New York, 1996

${ }^{2}$ S. J. Tans, A.R.M. Verschueren, C. Dekker, Nature 393 (1998) 49.

${ }^{3}$ H. Dai, N. Franklin and J. Han, Appl. Phys. Lett. 73 (1998) 1508.

${ }^{4}$ G.S. Duesberg, M. Burghard, J. Muster, G. Philipp, S. Roth, Chem. Comm. 3 (1998) 435.

${ }^{5}$ E. Richter and K. R. Subbaswamy, Phys. Rev. Lett. 79 (1997) 2738.

${ }^{6}$ R. Saito, T. Takeya, T. Kimura, G. Dresselhaus and M. S. Dresselhaus, Phys. Rev. B. 57 (1998) 4145.

${ }^{7}$ S. Bandow, S. Asaka, Y. Saito, A. M. Rao, L. Grigorian, E. Richter, P.C. Eklund, Phys. Rev. Lett. 80 (1998) 3779.

${ }^{8}$ A. Kasuya, Y. Sasaki, Y. Saito, K. Tohji, Y. Nishina, Phys. Rev. Lett. 72 (1997) 4434.

${ }^{9}$ J.M. Holden, P. Zhou, X.-X. Bi, P.C. Eklund, S. Bandow, R.A. Jishi, K. D.

Chowdhury, G. Dresselhaus, M. S. Dresselhaus, Chem. Phys. Lett. 220 (1994) 186.

${ }^{10}$ G.S. Duesberg, J. Muster, M. Burghard, S. Roth, H. Byrne, W. Blau, Synt. Met. in press

${ }^{11}$ A.M. Rao, E. Richter, S. Bandow, B. Chase, P.C. Eklund, K.A. Williams, S. Fang, K.R. Subbaswamy, M. Menon, A. Thess, R.E. Smalley, G. Dresselhaus, M.S. Dresselhaus, Science 75 (1997) 187.

${ }^{12}$ H. Kataura, Y. Kumazawa, Y. Maniwa, I. Umezu, S. Suzuki, Y. Ohtsuka, Y. Achiba, Synth. Met. in press

${ }^{13}$ M. Pimenta, A. Marucci, S.D.M. Brown M.J. Matthews, A. Rao, P.C. Eklund, R.E. Smalley, G. Dresselhaus, M.S. Dresselhaus, J. Mater. Res. 13 (1998) 2396.

${ }^{14}$ Z. K. Tang, H. D. Sun, J. Wang, J. Chen, G. Li, Appl. Phys. Lett. 73 (1998) 2287.

${ }^{15}$ O. Lourie, H.D. Wagner, J. Mater. Res. 13 (1998) 2418.

${ }^{16}$ S. Lefrant, M. Lamy de la Chapelle, I. Baltog, C. Journet, P. Bernier, E. Munoz, A. Benito, W.K. Maser, M.T. Martinez, G.F. de la Fuente, D. Laplaze, A. Loiseau, Mol. Cryst. Liq. Cryst. 322 (1998) 71.

${ }^{17}$ M. Lamy de la Chapelle, S. Lefrant, C. Journet, W. Maser, P. Bernier, Carbon 36 (1998) 705.

${ }^{18}$ C. Journet, W.K. Maser, P. Bernier, A. Loiseau, M. Lamy de la Chapelle, S. Lefrant, P. Deniard, R. Lee, J.E. Fischer, Nature 388 (1997) 756. 
${ }^{19}$ G.S. Duesberg, J. Muster. V. Krstic, M. Burghard, S. Roth, Appl. Phys. A 67 (1998) 117.

${ }^{20}$ F. Ni, T.M. Cotton, Anal. Chem. 58 (1986) 3159.

${ }^{21}$ M. Burghard, G.S. Duesberg, G. Philipp, J. Muster, S. Roth, Adv. Mater. 10 (1998) 584.

${ }^{22}$ M.S. Dresselhaus, G. Dresselhaus, Adv. Phys. 30, 139 (1981)

${ }^{23}$ A.M. Rao, E. Richter, S. Bandow, P.C. Eklund, Thin Solid Films 331, (1998) 141.

${ }^{24}$ T. Hertel, R.E. Walkup, P. Avouris, Phys. Rev. B. 58 (1998) 13870.

${ }^{25}$ G.S. Duesberg, to be published.

${ }^{26}$ R. Saito, G. Dresselhaus and M. S. Dresselhaus, Physical Properties of Carbon Nanotubes, Imperial College Press, London, (1998). 